\title{
An Improved Method for the Capillary Gas Chromatographic Derivatization of Polyhydroxylated Steroids Having tert-Hydroxyl Groups
}

\author{
Takashi IIDA, ${ }^{* \dagger}$ Keisuke ShIRAISHI, ${ }^{*}$ Shoujiro Ogawa, ${ }^{*}$ Junichi Goto, ${ }^{* *}$ and Toshio NaMbara** \\ *Department of Chemistry, College of Humanities \& Sciences, Nihon University, \\ Setagaya, Sakurajousui, Tokyo 156-8550, Japan \\ **Graduate School of Pharmaceutical Sciences, Tohoku University, Aobayama, Sendai 980-8578, Japan
}

\begin{abstract}
An improved method for a suitable derivatization of polyhydroxylated steroids having one or two tert-hydroxyl groups at the $5 \beta-, 14 \alpha-, 17 \alpha-, 24-$, and/or 25-positions by capillary gas chromatography (CGC) is described. By using trimethylsilyl triflate as a silylating reagent and 2,6-lutidine as a catalyst, each of $5 \beta$-cholane and $5 \alpha$-cholestane series of steroids was successfully transformed into trimethylsilyl (TMS) ether derivatives to give a single CGC peak under mild conditions. More bulky triethylsilyl (TES) etherification of $14 \alpha$ - and $17 \alpha$-hydroxy compounds provided multiple CGC peaks arising from completely- and/or incompletely-derivatized TES ethers accompanied by their thermal elimination products.
\end{abstract}

(Received April 14, 2003; Accepted June 23, 2003)

Recently, particular interest has been focused on an accurate and reliable method for capillary gas chromatographic (CGC) and gas chromatographic-mass spectrometric (GC-MS) analyses of tert-hydroxylated steroids. ${ }^{1}$ Since tert-hydroxyl groups in steroids are usually less reactive and sterically hindered, compared to prim- and sec-ones, and also prone to eliminate easily under acidic, alkaline or high temperature conditions, the development of their CGC and GC-MS measurements is a problem of great urgency. For these reasons, although a large number of CGC and GC-MS derivatizations and separations of prim- and sec-hydroxy steroids have been reported, ${ }^{2}$ analogous studies on tert-hydroxy steroids are severely limited.

We have previously reported the precolumn trimethylsilyl (TMS) and dimethylethylsilyl (DMES) etherifications of polyhydroxylated $5 \beta$-cholane and $5 \alpha$-cholestane series of steroids having one or two tert-hydroxyl groups in the steroid nucleus or the side chain. ${ }^{3}$ The precolumn derivatizing reagents involved were conventional reagents, such as hexamethyldisilazane/trimethylchlorosilane/pyridine (HMDS/TMCS/Py), $\quad \mathrm{N}, \mathrm{O}$-bis(trimethylsilyl)acetamide/ $N$ trimethylsilylimidazole/trimethylchlorosilane (BSA/TMSI/TMCS), and N,N-dimethylethylsilylimidazole (DMESI). Each of the resulting trialkylsilylation products afforded one to multiple CGC peaks arising from a fully- or partially-derivatized ether and/or their mixtures, depending upon the derivatizing reagents and reaction conditions employed. In addition to the derivatization procedures, the steric environment of the tert-hydroxyls (i.e., position and stereochemical conformation) in the substrates also strongly affected the degree of trialkylsilylations. A similar troublesome observation in TMS etherfication with HMDS/TMCS/Py or BSA/TMSI/TMCS has previously been reported for some ecdysteroids. ${ }^{4,5}$

\footnotetext{
$\doteqdot$ To whom correspondence should be addressed.

E-mail: takaiida@chs.nihon-u.ac.jp
}

The above intriguing findings in the CGC analysis of terthydroxylated steroids prompted us to investigate a more suitable and reliable CGC derivatization procedure for their analysis. This paper presents an improved method for the precolumn derivatization of a variety of structurally different polyhydroxylated bile acids and sterols containing one or two tert-hydroxyl groups.

\section{Experimental}

Materials

Almost all of the steroid samples were from our laboratory collection, which include new and natural tert-hydroxylated steroids recently synthesized in our laboratories. ${ }^{6,7}$ The silylating reagents, trimethylsilyl triflate and triethylsilyl triflate, and 2,6-lutidine, were available from Tokyo Kasei Kogyo Co., Ltd. (Tokyo, Japan). All other reagents and solvents used were of analytical reagent grade and used without further purification.

Derivatization of bile acid methyl esters and sterols with trialkylsilyl triflate

To a magnetically stirred solution of a steroid $(5 \mu \mathrm{g})$ dissolved in dry $\mathrm{CH}_{2} \mathrm{Cl}_{2}(300 \mu \mathrm{l})$ with ice-bath cooling, 2,6-lutidine (100 $\mu \mathrm{l})$ and then trialkylsilyl triflate $(50 \mu \mathrm{l})$ were added stepwise by a syringe, and the mixture was stirred at room temperature for 2 h. After the reaction, the organic layer was washed with water and dried with anhydrous $\mathrm{MgSO}_{4}$. An aliquot of the supernatant liquid was injected into $\mathrm{CGC}$, together with an internal reference compound $\left(\mathrm{C}_{38}\right)$.

\section{Gas chromatography}

A Model GC-17A gas chromatograph (Shimadzu, Kyoto, Japan) equipped with a flame ionization detector and a dataprocessing system (Chromatopac C-R6A) was employed. It 
was fitted with an Ultra ALLOY-1 (HT) stainless-steel capillary column $(30 \mathrm{~m} \times 0.25 \mathrm{~mm}$ i.d.; film thickness, $0.15 \mu \mathrm{m})$ (Frontier Lab. Ltd., Koriyama, Japan) coated with chemicallybonded and cross-linked non-polar dimethylsiloxane and operated under the following conditions: linear velocity of carrier gas (helium), $30 \mathrm{~cm} / \mathrm{s}$; make-up gas flow-rate, 60 $\mathrm{ml} / \mathrm{min}$; splitting ratio, 1:50; injection port, detector and column oven were maintained at 300,300 and $280^{\circ} \mathrm{C}$ for TMS ethers and 340,340 and $320^{\circ} \mathrm{C}$ for TES ethers, respectively.

\section{Gas chromatography-mass spectrometry}

Electron ionization-mass spectra (EI-MS) were obtained on a JEOL Model JMS-Automass 150 quadrupole mass spectrometry (Tokyo, Japan) interfaced to a Hewlett-Packerd Model 5890 Series III gas chromatograph. A cross-linked dimethylpolydisiloxane fused-silica capillary column (DB-1, 30 $\mathrm{m} \times 0.25 \mathrm{~mm}$ i.d.; film thickness, $0.25 \mu \mathrm{m})(\mathrm{J} \& \mathrm{~W}$ Scientific, Folsom, CA, USA) was inserted into the ion source through a direct inlet, and operated under the following conditions: ionization energy, $70 \mathrm{eV}$; emission current, $300 \mu \mathrm{A}$; mass range 4-1000 a.m.u.; injection port, column oven and ion source were kept at 280,300 and $180^{\circ} \mathrm{C}$, respectively.

\section{Results and Discussion}

Figure 1 shows the substrates examined in this study, which include a wide variety of $5 \beta$-cholane $(\mathbf{1 1 - 2 3}$; as the C-24 methyl ester) and $5 \alpha$-cholestane $(\mathbf{2 7 - 3 3 )}$ series of steroids possessing one or two tert-hydroxyl groups at the C-5, -14, -17, -24 , and/or -25 positions in the molecules. For a comparison, the CGC behavior of analogous bile acids $(\mathbf{1}-\mathbf{1 0})$ and sterols (24 - 26) having only several sec-hydroxyl groups at the C-3, -4, $-6,-7$ and/or -12 positions was also examined under the same derivatization and CGC conditions. Each of the derivatized CGC peaks was identified by GC-MS (see below).

It is well recognized that both prim- and sec-hydroxyl groups in steroids are readily transformed into their complete trialkylsilyl ether or acyl ester derivatives without difficulty. ${ }^{8}$ However, little is known about the reactivity of a tert-hydroxyl group, except for that of some ecdysteroids toward the silylation and esterification reagents. ${ }^{4,5}$ Thus, the precolumn CGC derivatization of ecdysteroids required a carefully controlled process, because of the different rates of derivatization, particularly for the position and stereochemical configuration of tert-hydroxyl groups present in the molecules. Meanwhile, we have recently revealed that the reactivity of tert-hydroxyl groups in steroids with HMDS/TMCS/Py, BSA/TMSI/TMCS and DMESI decreases in the following order: $25>24>5 \beta>$ $17 \alpha>14 \alpha .^{3}$

The use of acetic anhydride, trifluoroacetic anhydride, dimethyl-iso-propylsilylimidazole, $\quad \mathrm{N}$-methyl- $\mathrm{N}$-(tertbutyldimethylsilyl)-trifluoroacetamide or flophemesylchloride as common precolumn derivatizing reagents ${ }^{8}$ often gave multiple CGC peaks arising from a mixture of the fully- and partially-derivatized compounds.

Recently, successful use of trialkylsilyl trifluoromethanesulfonate (trialkylsilyl triflate, $\mathrm{CF}_{3} \mathrm{SO}_{3} \mathrm{SiR}_{3}$ ), a highly powerful silylating reagent and a strong Lewis acid in organic synthesis, ${ }^{9,10}$ for the regioselective derivatization of ouabain, ${ }^{11}$ prompted us to examine in detail the reagent in the precolumn CGC derivatization of compounds $\mathbf{1 1 - 2 3}$ and 27 33. Of various trialkylsilyl triflates, clean TMS etherification of the compounds was attained by using the least bulky trimethylsilyl triflate $\left[\mathrm{CF}_{3} \mathrm{SO}_{3} \mathrm{Si}\left(\mathrm{CH}_{3}\right)_{3}\right]$ in the presence of 2,6-

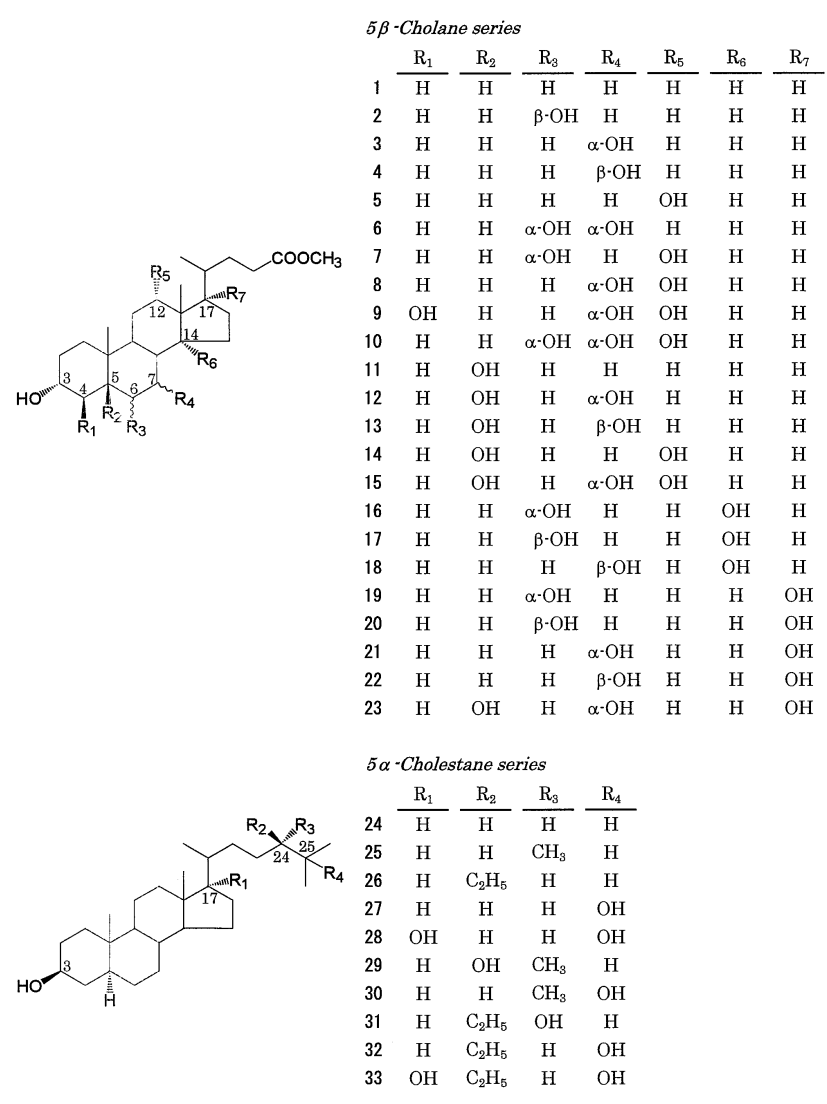

Fig. 1 Structures of the examined compounds.

lutidine in $\mathrm{CH}_{2} \mathrm{Cl}_{2}$ and afforded a single CGC peak with a theoretical shape, when each of the derivaization products was measured on a non-polar dimethylsiloxane column at $280^{\circ} \mathrm{C}$. The use of 2,6-lutidine as an alkaline catalyst was essential for TMS etherification. ${ }^{9}$ The retention data for compounds $\mathbf{1}-\mathbf{1 0}$ without tert-hydroxyls by trimethylsilyl triflate were in good agreement with those reported previously by HMDS/TMCS/Py reagent system..$^{2,3}$

TMS etherfication with trimethylsilyl triflate proceeded smoothly under mild conditions at room temperature for $2 \mathrm{~h}$. No multiple peaks arising from the acid or thermal elimination products were observed in each of the chromatograms (measured at $280^{\circ} \mathrm{C}$ ); in addition, ketene $O$-methyl $O$-trimethylsilyl acetals (silylenol ethers) or 2-trimethylsilylcarboxylates of the C-24 carboxylic acid esters in the side chain of $\mathbf{1}-\mathbf{2 3}$ were not also formed, ${ }^{10}$ as evidenced by the GC-MS analysis. An only exception was imperfect TMS etherification against compounds 16-18, in all of which $14 \alpha$-hydroxyl group was completely shielded ${ }^{3,5}$ to give the corresponding underivatized products.

The treatment of tert-hydroxylated steroids with more bulky triethylsilyl triflate $\left[\mathrm{CF}_{3} \mathrm{SO}_{3} \mathrm{Si}\left(\mathrm{CH}_{2} \mathrm{CH}_{3}\right)_{3}\right]$ and 2,6-lutidine also usually gave the corresponding complete TES ethers as a single product. As expected, the more bulky TES ethers were eluted much more slowly compared to the corresponding TMS ethers under the same CGC conditions, and separation of individual analogous isomers was much improved depending upon the number, position and stereochemical conformation of the hydroxyls (Fig. 2). However, the use of triethylsilyl triflate had several drawbacks. Thus, etherification product of $14 \alpha$-hydroxy compounds $(\mathbf{1 6}-\mathbf{1 8})^{3}$ gave a single peak, which was identified by GC-MS analysis as the respective $\Delta^{8(14)}$-unsaturated compounds. ${ }^{12}$ On the other hand, $17 \alpha$-hydroxy compounds (19- 
Table 1 Retention data for polyhydroxylated bile acids and sterols by trialkylsilylation with trialkylsilyl triflates

\begin{tabular}{|c|c|c|c|c|c|c|}
\hline \multirow{2}{*}{\multicolumn{2}{|c|}{ Hydroxyl subustituent }} & \multicolumn{2}{|c|}{ Trimethylsilylation } & \multicolumn{2}{|c|}{ Triethylsilylation } & \multirow{2}{*}{$\Delta[\mathrm{Um}]_{\mathrm{E}-\mathrm{M}}^{\mathrm{C}}$} \\
\hline & & \multirow[t]{2}{*}{$\mathrm{RRT}^{\mathrm{a}}$} & \multirow[t]{2}{*}{$\mathrm{MU}^{\mathrm{b}}$} & \multirow[t]{2}{*}{$\mathrm{RRT}^{\mathrm{a}}$} & $\mathrm{MU}^{\mathrm{b}}$ & \\
\hline \multicolumn{4}{|c|}{$5 \beta$-Cholane series } & & & \\
\hline 1 & $3 \alpha-\mathrm{OH}$ & 0.27 & 31.49 & 0.69 & 35.31 & 3.82 \\
\hline 2 & $3 \alpha, 6 \beta-(\mathrm{OH})_{2}$ & 0.31 & 32.27 & 1.15 & 39.04 & 6.77 \\
\hline 3 & $3 \alpha, 7 \alpha-(\mathrm{OH})_{2}$ & 0.32 & 32.32 & 1.17 & 39.05 & 6.73 \\
\hline 4 & $3 \alpha, 7 \beta-(\mathrm{OH})_{2}$ & 0.34 & 32.66 & 1.15 & 38.97 & 6.31 \\
\hline 5 & $3 \alpha, 12 \alpha-(\mathrm{OH})_{2}$ & 0.30 & 32.11 & 1.09 & 38.59 & 6.48 \\
\hline 6 & $3 \alpha, 6 \alpha, 7 \alpha-(\mathrm{OH})_{3}$ & 0.38 & 33.25 & 1.85 & 42.07 & 8.82 \\
\hline 7 & $3 \alpha, 6 \alpha, 12 \alpha-(\mathrm{OH})_{3}$ & 0.35 & 32.89 & 1.80 & 41.90 & 9.01 \\
\hline 8 & $3 \alpha, 7 \alpha, 12 \alpha-(\mathrm{OH})_{3}$ & 0.33 & 32.50 & 1.96 & 42.36 & 9.86 \\
\hline 9 & $3 \alpha, 4 \beta, 7 \alpha, 12 \alpha-(\mathrm{OH})_{4}$ & 0.50 & 34.70 & 3.34 & 45.62 & 10.92 \\
\hline 10 & $3 \alpha, 6 \alpha, 7 \alpha, 12 \alpha-(\mathrm{OH})_{4}$ & 0.38 & 33.31 & 3.15 & 45.28 & 11.97 \\
\hline 11 & $3 \alpha, 5 \beta-(\mathrm{OH})_{2}$ & 0.37 & 33.15 & 1.35 & 39.94 & 6.79 \\
\hline 12 & $3 \alpha, 5 \beta, 7 \alpha-(\mathrm{OH})_{3}$ & 0.38 & 33.25 & 2.00 & 42.33 & 9.08 \\
\hline 13 & $3 \alpha, 5 \beta, 7 \beta-(\mathrm{OH})_{3}$ & 0.42 & 33.73 & 1.93 & 42.12 & 8.39 \\
\hline 14 & $3 \alpha, 5 \beta, 12 \alpha-(\mathrm{OH})_{3}$ & 0.42 & 33.73 & 2.37 & 43.34 & 9.61 \\
\hline 15 & $3 \alpha, 5 \beta, 7 \alpha, 12 \alpha-(\mathrm{OH})_{4}$ & 0.39 & 33.37 & 3.54 & 45.63 & 12.26 \\
\hline 16 & $3 \alpha, 6 \alpha, 14 \alpha-(\mathrm{OH})_{3}$ & $\underline{0.30}$ & $\underline{31.97}$ & 1.05 & 38.43 & \\
\hline 17 & $3 \alpha, 6 \beta, 14 \alpha-(\mathrm{OH})_{3}$ & $\underline{0.29}$ & $\underline{31.81}$ & 1.05 & 38.43 & \\
\hline 18 & $3 \alpha, 7 \beta, 14 \alpha-(\mathrm{OH})_{3}$ & $\underline{0.28}$ & $\underline{31.56}$ & 0.96 & 37.76 & \\
\hline 19 & $3 \alpha, 6 \alpha, 17 \alpha-(\mathrm{OH})_{3}{ }^{\mathrm{d}}$ & $\overline{0.31}$ & 32.03 & $1.07 / 1.43 / 1.70$ & $38.52 / 40.48 / 41.60$ & 9.57 \\
\hline 20 & $3 \alpha, 6 \beta, 17 \alpha-(\mathrm{OH})_{3}{ }^{d}$ & 0.50 & 34.83 & $0.87 / 1.09 / 1.47 / 1.79$ & $36.88 / 38.51 / 40.49 / 41.78$ & \\
\hline 21 & $3 \alpha, 7 \alpha, 17 \alpha-(\mathrm{OH})_{3}{ }^{d}$ & 0.42 & 33.93 & $2.63 / 2.74 / 4.26$ & $. .44 .34 / 44.57 / \underline{47.25}$ & \\
\hline 22 & $3 \alpha, 7 \beta, 17 \alpha-(\mathrm{OH})_{3}{ }^{\mathrm{d}}$ & 0.32 & 32.46 & $0.97 / 1.06 / 1.71$ & $37.87 / 38.46 / 41.61$ & 9.15 \\
\hline 23 & $3 \alpha, 5 \beta, 7 \alpha, 17 \alpha-(\mathrm{OH})_{4}{ }^{d}$ & 0.30 & 32.20 & $2.64 / \underline{4.26}$ & $44.27 / \underline{47.19}$ & 12.07 \\
\hline \multicolumn{7}{|c|}{$5 \alpha$-Cholestane series } \\
\hline 24 & $3 \beta-\mathrm{OH}$ & 0.29 & 31.73 & 0.77 & 36.19 & 4.46 \\
\hline 25 & $(24 S)-24-\mathrm{Me}-3 \beta-\mathrm{OH}$ & 0.32 & 32.38 & 0.87 & 37.00 & 4.62 \\
\hline 26 & $(24 R)-24-\mathrm{Et}-3 \beta-\mathrm{OH}$ & 0.41 & 33.53 & 1.04 & 38.39 & 4.86 \\
\hline 27 & $3 \beta, 25-(\mathrm{OH})_{2}$ & 0.48 & 34.38 & 2.00 & 42.58 & 8.20 \\
\hline 28 & $3 \beta, 17 \alpha, 25-(\mathrm{OH})_{3}{ }^{d}$ & 0.54 & 35.00 & $1.46 / 1.62 / 3.31$ & $40.58 / 4.1 .25 / 45.67$ & 10.67 \\
\hline 29 & $(24 R)-24-\mathrm{Me}-3 \beta, 24-(\mathrm{OH})_{2}$ & 0.49 & 34.60 & 2.25 & 43.26 & 8.66 \\
\hline 30 & $(24 S)-24-\mathrm{Me}-3 \beta, 25-(\mathrm{OH})_{2}$ & 0.50 & 34.75 & 2.37 & 43.57 & 8.82 \\
\hline 31 & $(24 S)-24-\mathrm{Et}-3 \beta, 24-(\mathrm{OH})_{2}$ & 0.69 & 36.19 & 2.69 & 44.04 & 7.85 \\
\hline 32 & $(24 R)-24-\mathrm{Et}-3 \beta, 25-(\mathrm{OH})_{2}$ & 0.68 & 36.07 & 2.65 & 43.96 & 7.89 \\
\hline 33 & $(24 R)-24-\mathrm{Et}-3 \beta, 17 \alpha, 25-(\mathrm{OH})_{3}{ }^{\mathrm{d}}$ & 0.74 & 36.52 & $.1 .85 / 2.13 / 4.33$ & $41.72 / 42.56 / 46.57$ & 10.05 \\
\hline
\end{tabular}

a. Retention times relative to $\mathrm{C}_{38}$. b. Determined by using a mixture of even carbon number of C20 - C44 n-alkanes. c. Differences in the MU values between the fully-derivatized TMS and TES ether derivatives for the same compounds. d. Values underlined refer to the partially-derivatized silyl ethers, and values dotted underlined to the elimination products.

23, 28 and 33) by triethylsilyl triflate afforded multiple CGC peaks arising from a mixture of a partially- or fully-derivatized TES ethers, together with their respective $17 \alpha$-elimination products $\left(\Delta^{16}\right.$ and/or $\left.\Delta^{17(20)}\right)$ by GC-MS, ${ }^{10}$ probably owing to a steric hindrance and an elevated column temperature of $320^{\circ} \mathrm{C}$.

Table 1 compiles the CGC retention data for the examined compounds $(\mathbf{1}-\mathbf{3 3})$, which are expressed in terms of the relative retention times (RRT) and methylene unit (MU) values. The $\Delta[\mathrm{Um}]_{\mathrm{E}-\mathrm{M}}$ values, which are defined as the differences in the MU values between the completely-derivatized TMS and TES ethers for the same compounds, are also listed. The structures of each of the CGC peaks were confirmed by GC-MS. Table 2 gives the major fragment ions that appeared in the electron ionization-mass spectra (EI-MS) for the representative CGC peaks.

When the $\Delta[\mathrm{Um}]_{\mathrm{E}-\mathrm{M}}$ values were plotted against hydroxyl group number (Fig. 3), two regression lines with a good linearity, expressed as $y=2.62 x+1.33(n=18, r=0.967)$ and $y$ $=2.89 x+2.09(n=10, r=0.957)$, were obtained for $5 \beta$-cholane and $5 \alpha$-cholestane series of steroids, respectively. The data imply that the addition of one hydroxyl group in a steroid molecule produces $c a .2 .6-2.9$ units increases in the $\Delta[\mathrm{Um}]_{\mathrm{E}-\mathrm{M}}$ values, nearly corresponding to three methylene carbon increments, and that the number of hydroxyl groups present in

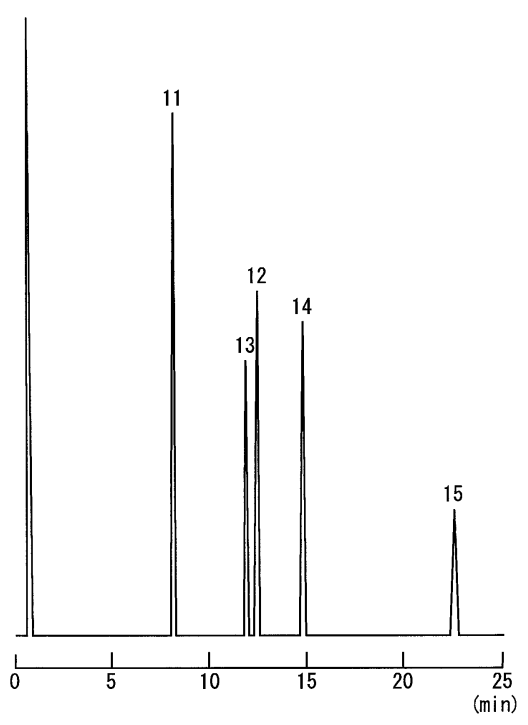

Fig. 2 Typical chromatogram of a mixture of isomeric $5 \beta$ hydroxylated bile acids (11-15) as their complete methyl ester-TES ethers on a non-polar dimethylsiloxane column measured at $320^{\circ} \mathrm{C}$. 
Table 2 GC-MS data for the TMS and TES etherification products of polyhydroxylated bile acids (as the C-24 methyl esters) and sterols

\begin{tabular}{|c|c|c|c|}
\hline $\begin{array}{l}\text { Position and stereochemistry } \\
\text { of hydroxyls }\end{array}$ & Derivatives formed & $\begin{array}{c}\text { MU } \\
\text { values }\end{array}$ & Major fragment ions above $\mathrm{m} / \mathrm{z}, 200$ (relative intensity, \%) \\
\hline \multicolumn{4}{|l|}{$5 \beta$-Cholane series } \\
\hline \multirow[t]{2}{*}{$3 \alpha, 7 \alpha, 12 \alpha-(\mathrm{OH})_{3}(\mathbf{8})$} & 3,7,12-trisTMS & 32.50 & $\begin{array}{l}638(\mathrm{M},<1 \%), 623\left(\mathrm{M}-\mathrm{CH}_{3}, 3 \%\right), 533,\left(\mathrm{M}-\mathrm{CH}_{3}-\mathrm{TMSOH}, 7 \%\right), 458(\mathrm{M}- \\
\text { 2TMSOH, 26\%), } 368 \text { (M-3TMSOH, 27\%), } 343 \text { (M-2TMSOH-S.C., 8\%), } \\
253 \text { (M-3TMSOH-S.C., 100\%) }\end{array}$ \\
\hline & 3,7,12-trisTES & 42.36 & 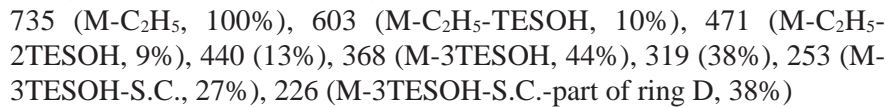 \\
\hline \multirow[t]{2}{*}{$3 \alpha, 5 \beta, 7 \beta-(\mathrm{OH})_{3}(\mathbf{1 3})$} & 3,5,7-trisTMS & 33.73 & $\begin{array}{l}638(\mathrm{M},<1 \%), 623\left(\mathrm{M}-\mathrm{CH}_{3},<1 \%\right), 548 \text { (M-TMSOH, 4\%), } 492(5 \%), \\
458 \text { (M-2TMSOH, 100\%), } 331(43 \%), 211 \text { (M-3TMSOH-S.C.-ring D, } \\
11 \%)\end{array}$ \\
\hline & 3,5,7-trisTES & 42.12 & $\begin{array}{l}735\left(\mathrm{M}-\mathrm{C}_{2} \mathrm{H}_{5}, 1 \%\right), 632(\mathrm{M}-\mathrm{TESOH}, 23 \%), 603\left(\mathrm{M}-\mathrm{C}_{2} \mathrm{H}_{5}-\mathrm{TESOH}, 55 \%\right), \\
500(\mathrm{M}-2 \mathrm{TESOH}, 100 \%), 457(21 \%), 368(\mathrm{M}-3 \mathrm{TESOH}, 50 \%), 326 \\
(27 \%), 272(14 \%)\end{array}$ \\
\hline \multirow[t]{2}{*}{$3 \alpha, 6 \alpha, 14 \alpha-(\mathrm{OH})_{3}(\mathbf{1 6})$} & 3,6-bisTMS-14-OH & 31.97 & $\begin{array}{l}548\left(\mathrm{M}-\mathrm{H}_{2} \mathrm{O},<1 \%\right), 533\left(\mathrm{M}-\mathrm{H}_{2} \mathrm{O}-\mathrm{CH}_{3}, 1 \%\right), 458\left(\mathrm{M}-\mathrm{H}_{2} \mathrm{O}-\mathrm{TMSOH},\right. \\
10 \%), 368\left(\mathrm{M}-\mathrm{H}_{2} \mathrm{O}-2 \mathrm{TMSOH}, 10 \%\right), 343\left(\mathrm{M}-\mathrm{H}_{2} \mathrm{O}-\mathrm{TMSOH}-\mathrm{S} . \mathrm{C} ., 22 \%\right), \\
253\left(\mathrm{M}-\mathrm{H}_{2} \mathrm{O}-2 \mathrm{TMSOH}-\mathrm{C} . \mathrm{C} ., 100 \%\right), 211\left(\mathrm{M}-\mathrm{H}_{2} \mathrm{O}-2 \mathrm{TMSOH}-\mathrm{S} . \mathrm{C} .-\mathrm{ring}\right. \\
\mathrm{D}, 11 \%)\end{array}$ \\
\hline & 3,6-bisTES- $\Delta^{8(14)}$ & 38.43 & 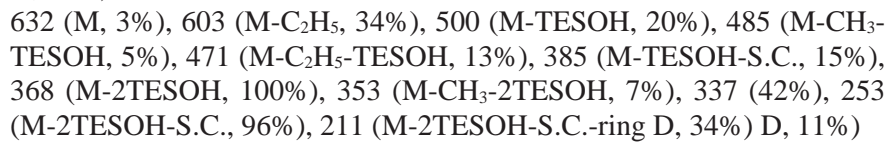 \\
\hline \multirow[t]{2}{*}{$3 \alpha, 6 \beta, 14 \alpha-(\mathrm{OH})_{3}(\mathbf{1 7})$} & 3,6-bisTMS-14-OH & 31.81 & $\begin{array}{l}548\left(\mathrm{M}-\mathrm{H}_{2} \mathrm{O},<1 \%\right), 533\left(\mathrm{M}-\mathrm{H}_{2} \mathrm{O}-\mathrm{CH}_{3}, 1 \%\right), 458\left(\mathrm{M}-\mathrm{H}_{2} \mathrm{O}-\mathrm{TMSOH},\right. \\
11 \%), 368\left(\mathrm{M}-\mathrm{H}_{2} \mathrm{O}-2 \mathrm{TMSOH}, 18 \%\right), 353\left(\mathrm{M}-\mathrm{H}_{2} \mathrm{O}-\mathrm{CH}_{3}-2 \mathrm{TMSOH}, 16 \%\right), \\
313\left(\mathrm{M}-\mathrm{H}_{2} \mathrm{O}-2 \mathrm{CH}_{3} \text {-TMSOH-S.C., 50\%), } 253\left(\mathrm{M}-\mathrm{H}_{2} \mathrm{O}-2 \mathrm{TMSOH}-\mathrm{C} . \mathrm{C} \text {, }\right.\right. \\
100 \%), 211\left(\mathrm{M}-\mathrm{H}_{2} \mathrm{O}-2 \mathrm{TMSOH}-\text { S.C.ring D, } 9 \%\right)\end{array}$ \\
\hline & 3,6-bisTES- $\Delta^{8(14)}$ & 38.43 & 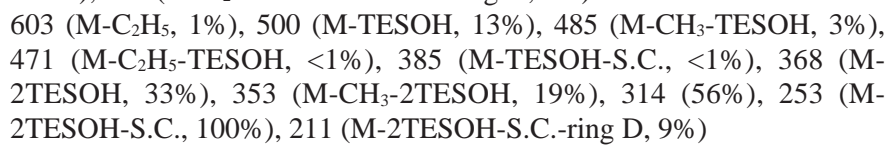 \\
\hline \multirow[t]{2}{*}{$3 \alpha, 7 \beta, 14 \alpha-(\mathrm{OH})_{3}(\mathbf{1 8})$} & 3,7-bisTMS-14-OH & 31.56 & $\begin{array}{l}548\left(\mathrm{M}-\mathrm{H}_{2} \mathrm{O},<1 \%\right), 533\left(\mathrm{M}-\mathrm{H}_{2} \mathrm{O}-\mathrm{CH}_{3}, 1 \%\right), 458\left(\mathrm{M}-\mathrm{H}_{2} \mathrm{O}-\mathrm{TMSOH}, 6 \%\right), \\
368\left(\mathrm{M}-\mathrm{H}_{2} \mathrm{O}-2 \mathrm{TMSOH}, 15 \%\right), 353\left(\mathrm{M}-\mathrm{H}_{2} \mathrm{O}-\mathrm{CH}_{3}-2 \mathrm{TMSOH}, 9 \%\right), 253 \\
\left(\mathrm{M}-\mathrm{H}_{2} \mathrm{O}-2 \mathrm{TMSOH}-\mathrm{S} . \mathrm{C} ., 100 \%\right), 239(22 \%)\end{array}$ \\
\hline & 3,7-bisTES- $\Delta^{8(14)}$ & 37.76 & $\begin{array}{l}603\left(\mathrm{M}-\mathrm{C}_{2} \mathrm{H}_{5}, 48 \%\right), 500 \text { (M-TESOH, 9\%), } 471\left(\mathrm{M}-\mathrm{C}_{2} \mathrm{H}_{5} \text {-TESOH, 31\%), }\right. \\
385 \text { (M-TESOH-S.C., } 8 \%), 368 \text { (M-2TESOH, 37\%), } 353\left(\mathrm{M}^{-\mathrm{CH}_{3}-}\right. \\
\text { 2TESOH, 15\%), } 337(15 \%), 253 \text { (M-2TESOH-S.C., 100\%), } 211(\mathrm{M}- \\
\text { 2TESOH-S.C.-ring D, } 12 \%)\end{array}$ \\
\hline \multirow[t]{3}{*}{$3 \alpha, 6 \alpha, 17 \alpha-(\mathrm{OH})_{3}(\mathbf{1 9})$} & 3,6,17-trisTMS & 32.03 & 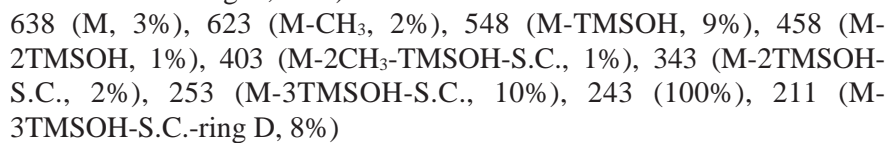 \\
\hline & $\begin{array}{l}\text { 3,6-bisTES- } \Delta^{16} \\
\left(\text { or } \Delta^{17(20)}\right)\end{array}$ & 38.52 & $\begin{array}{l}\left.603\left(\mathrm{M}_{-} \mathrm{C}_{2} \mathrm{H}_{5},<1 \%\right), 517 \text { (M-S.C., } 12 \%\right), 471\left(\mathrm{M}-\mathrm{C}_{2} \mathrm{H}_{5}-\mathrm{TESOH}, 1 \%\right), \\
385(\mathrm{M}-\mathrm{TESOH}-\mathrm{S} . \mathrm{C} ., 15 \%), 339\left(\mathrm{M}-\mathrm{C}_{2} \mathrm{H}_{5}-2 \mathrm{TESOH}, 3 \%\right), 319(3 \%), 253 \\
\text { (M-2TESOH-S.C., } 100 \%), 211 \text { (M-2TESOH-S.C.-ring D, 18\%) }\end{array}$ \\
\hline & 3,6,17-trisTES & 41.60 & 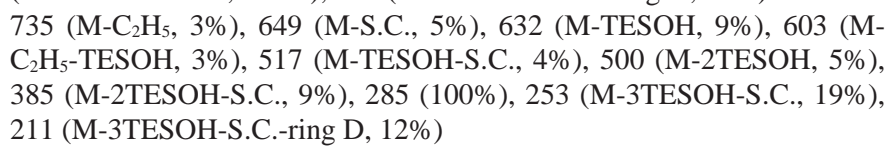 \\
\hline \multirow[t]{3}{*}{$3 \alpha, 7 \beta, 17 \alpha-(\mathrm{OH})_{3}(\mathbf{2 2})$} & 3,7,17-trisTMS & 32.46 & 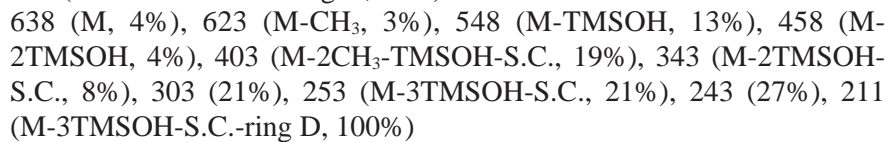 \\
\hline & $\begin{array}{l}\text { 3,7-bisTES- } \Delta^{16} \\
\left.\text { (or } \Delta^{17(20)}\right)\end{array}$ & 37.87 & $\begin{array}{l}\left.632(\mathrm{M}<1 \%), 603\left(\mathrm{M}_{-} \mathrm{C}_{2} \mathrm{H}_{5}, 2 \%\right), 517 \text { (M-S.C., } 38 \%\right), 471\left(\mathrm{M}-\mathrm{C}_{2} \mathrm{H}_{5-}^{-}\right. \\
\left.\text {TESOH, } 1 \%), 385 \text { (M-TESOH-S.C., } 8 \%), 339 \text { (M-C } \mathrm{C}_{2} \mathrm{H}_{5}-2 \mathrm{TESOH}, 3 \%\right) \text {, } \\
319(3 \%), 253 \text { (M-2TESOH-S.C., 100\%), } 211 \text { (M-2TESOH-S.C.-ring D, } \\
11 \%)\end{array}$ \\
\hline & 3,7,17-trisTES & 41.61 & 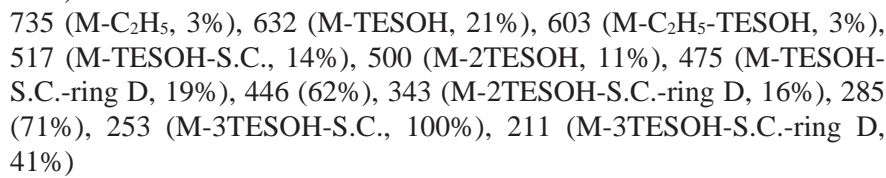 \\
\hline \multirow{2}{*}{$\begin{array}{l}5 \alpha \text {-Cholestane series } \\
(24 \mathrm{~S})-24-\mathrm{Me}-3 \beta \text {-OH (25) }\end{array}$} & 3-TMS & 32.38 & $\begin{array}{l}474(\mathrm{M}, 9 \%), 459\left(\mathrm{M}-\mathrm{CH}_{3}, 7 \%\right), 369\left(\mathrm{M}^{-\mathrm{CH}_{3}-\mathrm{TMSOH}}, 18 \%\right), 323 \\
(11 \%), 253(\mathrm{M}-\mathrm{TMSOH}-\mathrm{S} . \mathrm{C} ., 100 \%), 209(13 \%)\end{array}$ \\
\hline & 3-TES & 37.00 & $\begin{array}{l}\left.516(\mathrm{M}, 32 \%), 487\left(\mathrm{M}-\mathrm{C}_{2} \mathrm{H}_{5}, 5 \%\right), 384 \text { (M-TESOH, } 28 \%\right), 369\left(\mathrm{M}-\mathrm{CH}_{3}-\right. \\
\text { TESOH, 100\%), } 342(74 \%), 257 \text { (M-TESOH-S.C., 54\%), } 215(\mathrm{M}- \\
\text { TESOH-S.C.-ring D, 69\%) }\end{array}$ \\
\hline
\end{tabular}




\begin{tabular}{|c|c|c|c|}
\hline \multirow[t]{2}{*}{$3 \beta, 25-(\mathrm{OH})_{2}(27)$} & 3,25-bisTMS & 34.38 & $\begin{array}{l}548(\mathrm{M}, 6 \%), 533\left(\mathrm{M}-\mathrm{CH}_{3}, 73 \%\right), 458(\mathrm{M}-\mathrm{TMSOH}, 28 \%), 443\left(\mathrm{M}-\mathrm{CH}_{3}-\right. \\
\mathrm{TMSOH}, 42 \%), 353\left(\mathrm{M}-\mathrm{CH}_{3}-2 \mathrm{TMSOH}, 21 \%\right), 347 \text { (M-TMSOH-S.C., } \\
22 \%), 305(18 \%), 257(\mathrm{M}-2 \mathrm{TMSOH}-\mathrm{S} . \mathrm{C} ., 40 \%), 230(18 \%), 215 \text { (M- } \\
\text { 2TMSOH-S.C.-ring D, 100\%) }\end{array}$ \\
\hline & 3,25-bisTES & 42.58 & $\begin{array}{l}617\left(\mathrm{M}-\mathrm{CH}_{3}, 1 \%\right), 603\left(\mathrm{M}-\mathrm{C}_{2} \mathrm{H}_{5}, 100 \%\right), 500(\mathrm{M}-\mathrm{TESOH}, 9 \%), 471\left(\mathrm{M}-{ }^{-}\right. \\
\mathrm{C}_{2} \mathrm{H}_{5} \text {-TESOH, 42\%), } 368(\mathrm{M}-2 \mathrm{TESOH}, 7 \%), 316(7 \%), 257 \text { (M- } \\
\text { 2TESOH-S.C., 7\%), } 205(20 \%)\end{array}$ \\
\hline \multirow[t]{2}{*}{$3 \beta, 17 \alpha, 25-(\mathrm{OH})_{3}(\mathbf{2 8})$} & 3,17,25-trisTMS & 35.00 & $\begin{array}{l}636(\mathrm{M}, 3 \%), 621\left(\mathrm{M}-\mathrm{CH}_{3}, 3 \%\right), 531\left(\mathrm{M}-\mathrm{CH}_{3}-\mathrm{TMSOH}, 2 \%\right), 490(17 \%), \\
435 \text { (M-TMSOH-S.C., 100\%), } 345 \text { (M-2TMSOH-S.C., 9\%), } 255 \text { (M- } \\
\text { 3TMSOH-S.C., } 37 \%), 239(73 \%)\end{array}$ \\
\hline & 3,17,25-trisTES & 45.67 & 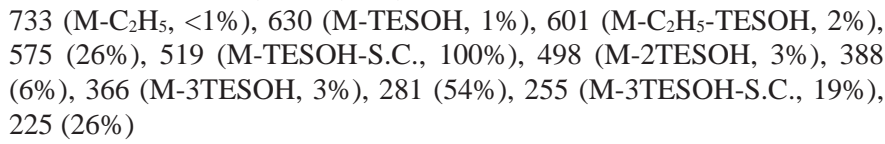 \\
\hline \multirow[t]{2}{*}{ (24R)-24-Me-3 $\beta, 24-(\mathrm{OH})_{2}$ (29) } & 3,24-bisTMS & 34.60 & $\begin{array}{l}547\left(\mathrm{M}_{-} \mathrm{CH}_{3}, 30 \%\right), 519\left(\mathrm{M}-\mathrm{CH}\left(\mathrm{CH}_{3}\right)_{2}, 94 \%\right), 457\left(\mathrm{M}-\mathrm{CH}_{3}-\mathrm{TMSOH},\right. \\
5 \%), 429\left(\mathrm{M}-\mathrm{CH}\left(\mathrm{CH}_{3}\right)_{2}-\mathrm{TMSOH}, 30 \%\right), 339\left(\mathrm{M}-\mathrm{CH}\left(\mathrm{CH}_{3}\right)_{2}-2 \mathrm{TMSOH},\right. \\
100 \%), 283(23 \%), 257 \text { (M-2TMSOH-S.C., 55\%), } 215(\mathrm{M}-2 \mathrm{TMSOH}- \\
\text { S.C.-ring D, } 49 \%)\end{array}$ \\
\hline & 3,24-bisTES & 43.26 & $\begin{array}{l}617\left(\mathrm{M}-\mathrm{C}_{2} \mathrm{H}_{5}, 11 \%\right), 603\left(\mathrm{M}-\mathrm{CH}\left(\mathrm{CH}_{3}\right)_{2}, 17 \%\right), 514(\mathrm{M}-\mathrm{TESOH}, 3 \%) \text {, } \\
485\left(\mathrm{M}-\mathrm{C}_{2} \mathrm{H}_{5}-\mathrm{TESOH}, 8 \%\right), 382(\mathrm{M}-2 \mathrm{TESOH}, 2 \%), 339\left(\mathrm{M}-\mathrm{CH}\left(\mathrm{CH}_{3}\right)_{2}-\right. \\
\text { 2TESOH, } 4 \%), 200\left(\mathrm{M}-\mathrm{CH}_{3}-2 \mathrm{TESOH}-\mathrm{S} . \mathrm{C} .- \text { ring D, 100\%) }\right.\end{array}$ \\
\hline
\end{tabular}

a. M, molecular ion; TMSOH, trimethylsilanol (90 a.m.u.); TESOH, triethylsilanol (132 a.m.u.); S.C., side chain (C ${ }_{6} \mathrm{H}_{11} \mathrm{O}_{2}, 115$ a.m.u.; $\mathrm{C}_{8} \mathrm{H}_{17}, 113$ a.m.u.; $\mathrm{C}_{9} \mathrm{H}_{19}, 127$ a.m.u.; $\mathrm{C}_{10} \mathrm{H}_{21}, 141$ a.m.u.); ring D (42 a.m.u.); part of ring D (27 a.m.u.).

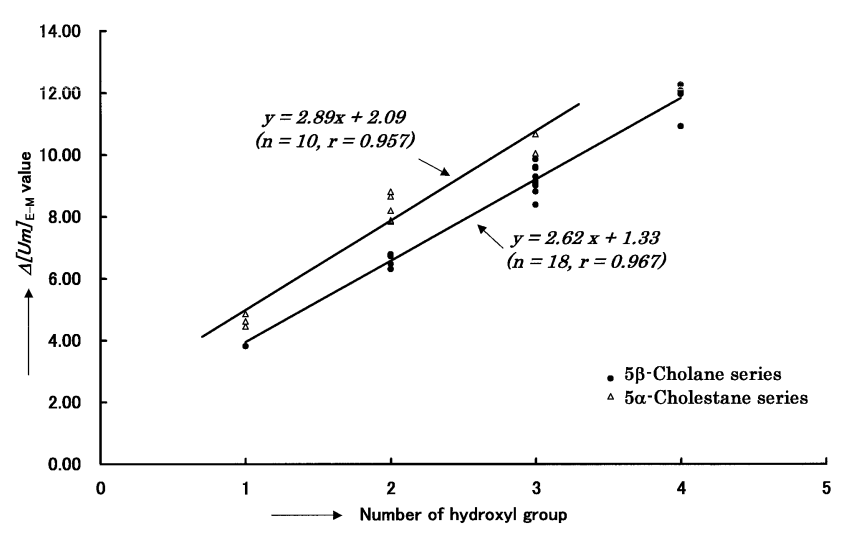

Fig. 3 Relationship between $\Delta[\mathrm{Um}]_{\mathrm{E}-\mathrm{M}}$ values and the number of hydroxyl groups in polyhydroxylated $5 \beta$-cholane and $5 \alpha$-cholestane series of steroids. Data were obtained from the differences in the MU values between the fully-derivatized TMS and TES ethers for the same compounds; linear regression lines and correlation coefficients ( $r$ ) for $5 \beta$-cholane and $5 \alpha$-cholestane series of steroids were individually calculated from the respective $\Delta[\mathrm{Um}]_{\mathrm{E}-\mathrm{M}}$ values.

the substrates can be deduced from the regression equations. In other words, if the observed $\Delta[\mathrm{Um}]_{\mathrm{E}-\mathrm{T}}$ value of the CGC peak in an unknown steroid deviates considerably from the regression line, it must possess a free tert-hydroxyl group or its elimination product. Particular attention should, therefore, be paid for $14 \alpha$ and $17 \alpha$-hydroxylated steroids, as mentioned above.

In conclusion, a suitable CGC derivatization was developed for a wide variety of polyhydroxylated steroids containing terthydroxyl groups. The method, which involves the combined use of trialkylsilyl triflate (especially trimethylsilyl triflate) and 2,6-lutidine, may be of wide utility for the determination of secand tert-hydroxylated steroids.

\section{References}

1. H. R. Lamparczyk, "CRC Handbook of Chromatography: Analysis and Characterization of Steroids", ed. J. Sherma, 1992, CRC Press, Boca Raton, Ann Arbor, London, Tokyo.

2. T. Iida, T. Momose, T. Tamura, T. Matsumoto, J. Goto, T. Nambara, and F. C. Chang, J. Chromatogr., 1987, 389, 155 .

3. T. Iida, M. Hikosaka, J. Goto, and T. Nambara, J. Chromatogr. A, 2001, 937, 97.

4. C. R. Bieby, E. D. Morgan, and I. D. Wilson, J. Chromatogr., 1986, 351, 57.

5. R. P. Evershed, J. G. Mercer, and H. H. Rees, J. Chromatogr., 1987, 390, 357.

6. T. Iida, T. Yamaguchi, R. Nakamori, M. Hikosaka, N. Mano, J. Goto, and T. Nambara, J. Chem. Soc. Perkin Trans. 1, 2001, 2229.

7. T. Iida, K. Shiraishi, S. Ogawa, T. Goto, N. Mano, J. Goto, and T. Nambara, Lipids, 2003, 38, 191.

8. K. Blau and M. Halket (ed.), "Handbook of Derivatives for Chromatography", 1993, John Wiley \& Sons., Chichester, England.

9. E. J. Corey, H. Cho, C. Rücker, and D. H. Hua, Tetrahedron Lett., 1981, 22, 3455.

10. H. Emde, D. Domsch, H. Feger, U. Frick, A. Götz, H. H. Hergott, K. Hofmann, W. Kober, K. Krägeloh, T. Oesterle, W. Steppan, W. West, and G. Simchen, Synthesis, 1982, 1.

11. J. F. Templeton, J. M. Hamlyn, B. P. Hamilton, J. Ayotte, H. Majgier-Baranowska, A. Lester, K. Marat, and H. Perreault, Steroids, 2000, 65, 379.

12. H. Budzikiewicz, "Biomedical Applications of Mass Spectrometry”, ed. G. R. Waller, 1972, Wiley-Interscience, New York. 